\title{
JOGO EDUCATIVO PARA A PREVENÇÃO DA ALIENAÇÃo PARENTAL DE CRIANÇAS E ADOLESCENTES
}

\section{EDUCATIONAL GAME TO PREVENT PARENTAL ALIENATION OF CHILDREN AND ADOLESCENTS}

\author{
JUEGO EDUCATIVO PARA PREVENIR LA ALIENACIÓN DE NIÑOS Y ADOLESCENTES POR LOS \\ PADRES
}

Maria Aparecida Beserra¹, Emily Roberta Gonçalves da Silva², Gabriela Guimarães da Mota ${ }^{3}$, Gabriela Wanderley da Silva ${ }^{4}$, Gabrielle Valeriano de Albuquerque ${ }^{5}$, João Vitor do Nascimento Olímpio ${ }^{6}$, Fernanda Jorge Magalhães ${ }^{7}$, Claudinalle Farias Queiroz de Souza ${ }^{8}$, Walmir Soares da Silva Junior ${ }^{9}$

Submetido em: 07/07/2021

e27563 Aprovado em: 10/08/20021

https://doi.org/10.47820/recima21.v2i7.563

\section{RESUMO}

Objetivo: Relatar o desenvolvimento de uma tecnologia educativa para crianças e adolescentes no formato de game direcionado à alienação parental como intervenção de Enfermagem. Método: Estudo do tipo relato de experiência. Foi desenvolvido por discentes e docentes de uma Universidade Pública de Pernambuco, durante a experiência do ensino a distância, de março a abril de 2021. Respeitou as diretrizes da Resolução $n^{\circ}$ 466/2012. Para construção utilizou-se da proposta em etapas do design thinking, por meio do levantamento bibliográfico, desenvolvimento das etapas do design do game, elaboração do game propriamente dito e apresentação. Resultados: A produção do game "AlieNÃO" proporcionou aos acadêmicos uma ampliação de seus conhecimentos acerca das ferramentas tecnológicas, bem como a descoberta de instrumentos que podem ser utilizados para educação em saúde, visando enquadrar essa metodologia ao contexto da atualidade e atingir o máximo de usuários. Considerações finais: $O$ jogo educativo desenvolvido é um método atual e inovador que pode ser utilizado como um recurso didático tecnológico acessível para uso em escolas e unidades de saúde para discussão sobre esse agravo.

PALAVRAS-CHAVE: Informática em Enfermagem. Direitos da criança e do adolescente. Jogos de vídeo. Tecnologia educacional.

\section{ABSTRACT}

Objective: To report the development of an educational technology for children and adolescents in a game format aimed at parental alienation as a Nursing intervention. Method: Study of the experience report type. It was developed by students and professors of a Public University of Pernambuco, during the distance learning experience, from March to April 2021. It complied with the guidelines of Resolution No. 466/2012. For construction, the proposal was used in stages of design thinking, through a bibliographic survey,

\footnotetext{
1 Doutora. Enfermeira Docente do Curso de Bacharelado de Enfermagem da Faculdade de Enfermagem Nossa Senhora das Graças (FENSG) da Universidade de Pernambuco (UPE).

${ }^{2}$ Acadêmica do Curso de Bacharelado de Enfermagem da Faculdade de Enfermagem Nossa Senhora das Graças (FENSG) da Universidade de Pernambuco (UPE).

${ }^{3}$ Acadêmica do Curso de Bacharelado de Enfermagem da Faculdade de Enfermagem Nossa Senhora das Graças (FENSG) da Universidade de Pernambuco (UPE).

${ }^{4}$ Acadêmica do Curso de Bacharelado de Enfermagem da Faculdade de Enfermagem Nossa Senhora das Graças (FENSG) da Universidade de Pernambuco (UPE).

${ }^{5}$ Acadêmica do Curso de Bacharelado de Enfermagem da Faculdade de Enfermagem Nossa Senhora das Graças (FENSG) da Universidade de Pernambuco (UPE).

${ }^{6}$ Acadêmico do Curso de Bacharelado de Enfermagem da Faculdade de Enfermagem Nossa Senhora das Graças (FENSG) da Universidade de Pernambuco (UPE).

7 Doutora. Enfermeira Docente do Curso de Bacharelado de Enfermagem da Faculdade de Enfermagem Nossa Senhora das Graças (FENSG) da Universidade de Pernambuco (UPE)

8 Doutora. Enfermeira Docente do Curso de Bacharelado de Enfermagem da Faculdade de Enfermagem Nossa Senhora das Graças (FENSG) da Universidade de Pernambuco (UPE)

9 Mestre. Analista de Sistemas Docente do Curso de Bacharelado de Enfermagem da Faculdade de Enfermagem Nossa Senhora das Graças (FENSG) da Universidade de Pernambuco (UPE)

RECIMA21 - Ciências Exatas e da Terra, Sociais, da Saúde, Humanas e Engenharia/Tecnologia
} 


\title{
RECIMA21 - REVISTA CIENTÍFICA MULTIDISCIPLINAR ISSN 2675-6218
}

\author{
JOGO EDUCATIVO PARA A PREVENÇÃO DA ALIENAÇÃO PARENTAL DE CRIANÇAS E ADOLESCENTES \\ Maria Aparecida Beserra, Emily Roberta Gonçalves da Silva, Gabriela Guimarães da Mota, \\ Gabriela Wanderley da Silva, Gabrielle Valeriano de Albuquerque, João Vitor do Nascimento Olímpio, \\ Fernanda Jorge Magalhães, Claudinalle Farias Queiroz de Souza, Walmir Soares da Silva Junior
}

preparation of the game design, development of the game itself and evaluation. Results: The production of the game "AlieNÃ̂" provided academics with an expansion of their knowledge about technological tools, as well as the discovery of instruments that can be used for health education, aiming to fit this methodology to the current context and achieve the maximum of users. Final considerations: The educational game developed is a current and innovative method that can be used as an accessible technological didactic resource for use in schools and health units to discuss this problem.

KEYWORDS: Nursing Informatics. Child Advocacy. Video Games. Educational Technology.

\section{RESUMEN}

Objetivo: Informar el desarrollo de una tecnología educativa para niños y adolescentes en formato de juego orientado a la alienación parental como intervención de Enfermería. Método: Estudio del tipo de relato de experiencia. Fue desarrollado por estudiantes y profesores de una Universidad Pública de Pernambuco, durante la experiencia de aprendizaje a distancia, de marzo a abril de 2021. Se cumplió con los lineamientos de la Resolución No. 466/2012. Para la construcción, la propuesta se utilizó en etapas de pensamiento de diseño, a través de un relevamiento bibliográfico, elaboración del diseño del juego, desarrollo del propio juego y evaluación. Resultados: La producción del juego "AlieNÃO" brindó a los académicos una expansión de sus conocimientos sobre herramientas tecnológicas, así como el descubrimiento de instrumentos que pueden ser utilizados para la educación en salud, con el objetivo de adecuar esta metodología al contexto actual y lograr el máximo de usuarios. Consideraciones finales: El juego educativo desarrollado es un método actual e innovador que puede ser utilizado como un recurso didáctico tecnológico accesible para ser utilizado en escuelas y unidades de salud para discutir esta problemática.

PALABRAS CLAVES: Informática de enfermería. Defensa de la infancia. Juegos de vídeo. Tecnologia Educacional

\section{INTRODUÇÃO}

A infância e adolescência são períodos que apresentam intensas mudanças físicas, psicológicas e comportamentais cujo desenvolvimento racional e emocional pode ser marcado por constantes aprendizados e descobertas. Nestas fases, a família tem papel de destaque no que se refere aos cuidados oferecidos às crianças e adolescentes. Para isso, é importante que os adultos compreendam esses indivíduos em seu contexto como pessoas, em família e nas suas atividades diárias em comunidade, para que possam transmitir segurança e confiança, além de promover um ambiente em que eles consigam se expressar e se desenvolver em sua plenitude de forma saudável e segura (PORTO; DUPONT, 2016).

Com o objetivo de garantir um ambiente familiar saudável, a Lei № 12.318 de 2010 dispõe sobre a Alienação Parental, está definida como um ato de interferência na formação psicológica da criança ou do adolescente promovida ou induzida por um dos genitores, avós ou pelos que tenham o menor sob sua autoridade, guarda ou vigilância, para provocar repúdio ao genitor não-guardião ou causar danos no vínculo com ele (BRASIL, 2010).

Tal lei foi escrita com base na Síndrome de Alienação Parental (SAP). Esta foi descrita pelo psiquiatra forense norte-americano Richard Gardner como uma perturbação da infância ou adolescência no contexto de separação conjugal e cuja manifestação seria uma campanha feita por um dos pais para a criança denegrir, rejeitar e odiar o outro genitor. Contudo, a lei não trata a alienação parental como patologia, mas sim como uma conduta que merece intervenção judicial (MONTEZUMA; PEREIRA; MELO, 2017). 


\section{RECIMA21 - REVISTA CIENTÍFICA MULTIDISCIPLINAR ISSN 2675-6218}

JOGo EDUCATIVO PARA A PREVENÇÃo DA ALIENAÇÃo PARENTAL DE CRIANÇAS E ADOLESCENTES Maria Aparecida Beserra, Emily Roberta Gonçalves da Silva, Gabriela Guimarães da Mota, Gabriela Wanderley da Silva, Gabrielle Valeriano de Albuquerque, João Vitor do Nascimento Olímpio, Fernanda Jorge Magalhães, Claudinalle Farias Queiroz de Souza, Walmir Soares da Silva Junior

No atual cenário brasileiro o crime de alienação parental vem ganhando notoriedade devido, sobretudo, ao aumento de casos de divórcios e disputas judiciais pela guarda dos filhos (IBGE, 2017). Estas situações são apontadas como indícios de alienação parental devido à separação hostil, que pode gerar conflitos entre os cônjuges e refletir diretamente, de forma negativa nos filhos. Por esse motivo, a fim de evitar sequelas no crescimento e desenvolvimento de crianças e adolescentes, é importante que a situação seja identificada precocemente para que possa ser feita uma intervenção especializada e multidimensional com todos os envolvidos (PEREIRA AIRES, 2018).

Compreendendo esse contexto e com o propósito de alcançar a divulgação de tal condição social e de saúde junto às crianças, adolescentes e seus familiares/cuidadores, o enfermeiro pode atuar como ponte de conhecimento e utilizar-se de recursos tecnológicos que facilitem o acesso à informação.

Destaca-se assim, a gamificação como metodologia que pode ser empregada para a apoiar a identificação, conhecimento e discussão de tal problemática. Esta consiste em uma técnica provinda dos games com aplicação em contextos alternativos, com intenção de se obter a solução do problema, engajamento dos usuários e melhores resultados no contexto em que é aplicada (SILVA, 2019). Essa abordagem apresenta-se como um benefício para os dias atuais, em que os indivíduos estão cada vez mais inseridos em contextos de mídias digitais, redes sociais, tecnologias integrativas, o que favorece a promoção de intervenções em saúde (POSSOLLI; MARCHIORATO; NASCIMENTO, 2020).

Esse aumento de interação das pessoas com as Tecnologias da Informação e Comunicação (TIC) tem impactado no processo de trabalho em Saúde e Enfermagem. Dessa forma, o enfermeiro, com o intuito de aprimorar constantemente os cuidados oferecidos às crianças e aos adolescentes, pode utilizarse da gamificação como uma estratégia para promover a educação em saúde para esses indivíduos e suas famílias/cuidadores, beneficiando e inovando a aprendizagem acerca da alienação parental (CASTRO; GONÇALVES, 2018).

Diante do exposto, o objetivo deste estudo foi relatar o desenvolvimento de uma tecnologia educativa para crianças e adolescentes no formato de game direcionado à alienação parental como intervenção de Enfermagem.

\section{METODOLOGIA}

O estudo é do tipo relato de experiência, desenvolvido entre os meses de março e abril de 2021, na Unidade Temática (UT) Informatização em Saúde do $5^{\circ}$ módulo do curso de Bacharelado em Enfermagem, de uma Universidade pública localizada no Nordeste do Brasil, no período de março a abril de 2021.

Foi desenvolvido mediante um "Serious Games" produzido na plataforma online Scratch. Plataforma que compreende um projeto utilitário online e open acess, de linguagem visual de padrão de codificação, que se sobressai por ser um excelente recurso para o ensino de suporte lógico de programação por possuir os principais suportes de um sistema, como: variáveis, operadores, estruturas de decisão e repetição e outros, de maneira simplificada e criativa, ajudando os programadores a raciocinar de forma sistemática e a trabalhar cooperativamente (SCRATCH, 2013). 


\section{RECIMA21 - REVISTA CIENTÍFICA MULTIDISCIPLINAR ISSN 2675-6218}

JOGO EDUCATIVO PARA A PREVENÇÃo DA ALIENAÇÃo PARENTAL DE CRIANÇAS E ADOLESCENTES Maria Aparecida Beserra, Emily Roberta Gonçalves da Silva, Gabriela Guimarães da Mota, Gabriela Wanderley da Silva, Gabrielle Valeriano de Albuquerque, João Vitor do Nascimento Olímpio, Fernanda Jorge Magalhães, Claudinalle Farias Queiroz de Souza, Walmir Soares da Silva Junior

O procedimento realizado seguiu a estratégia do design thinking utilizando-se das fases de ideação, imersão e prototipação. Na fase de imersão, houve a elaboração da ideia do game associando aos objetivos educacionais do módulo. A fase de imersão utilizou-se da construção de competências no campo prevenção de agravos na Enfermagem no tema, utilizando-se da revisão sistemática. E a fase de prototipação, foi propriamente a criação do protótipo do jogo no Scratch, no qual foi escolhido seu formato, construção das cenas, minijogos e forma de abordagem do conteúdo, produção do jogo na plataforma.

A partir da assimilação do tema, foi-se traçada a sistematização do jogo seguindo as seguintes etapas: levantamento bibliográfico, elaboração do jogo, designer do jogo e fase de avaliação.

O levantamento bibliográfico se deu por meio da realização da pesquisa nas bases de dados Scientific Electronic Library Online - SciELO, Acervo Digital da UFRGS e na Biblioteca Virtual em Saúde BVS, além da consulta no ECA, Constituição Federal (CF) e Instituto Brasileiro de Geografia e Estatística (IBGE). Foram utilizados os seguintes descritores em língua portuguesa: "Criança e Adolescente", "Alienação Parental", "Saúde", "Síndrome da Alienação Parental" com o operador boleano "OR" e recorte temporal dos últimos 10 anos (2010-2020). O processo de seleção dos estudos se deu por meio da declaração de itens de relatório preferidos para revisões sistemáticas e meta-análises (PRISMA), totalizando uma amostra final de quatro estudos (Figura 1).

Figura 1. Fluxograma PRISMA da revisão sistemática na fase do levantamento de dados. Recife, 2021.

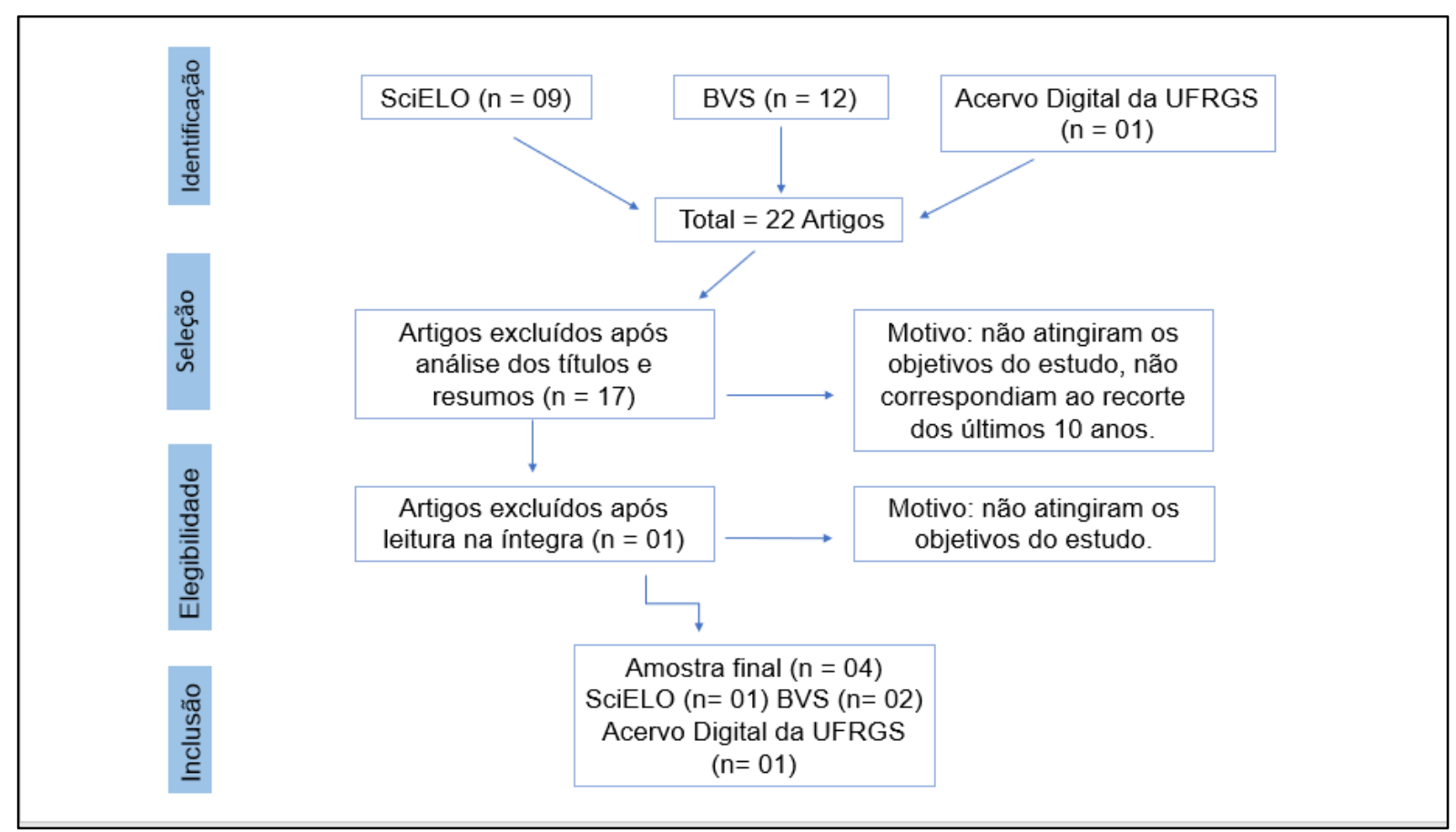

Fonte: Autores.

Para a elaboração do jogo alguns componentes como dinâmica familiar e situações corriqueiras da SAP foram selecionados a partir da leitura do referencial teórico selecionado no levantamento 


\section{RECIMA21 - REVISTA CIENTÍFICA MULTIDISCIPLINAR ISSN 2675-6218}

JOGO EDUCATIVO PARA A PREVENÇÃO DA ALIENAÇÃO PARENTAL DE CRIANÇAS E ADOLESCENTES Maria Aparecida Beserra, Emily Roberta Gonçalves da Silva, Gabriela Guimarães da Mota, Gabriela Wanderley da Silva, Gabrielle Valeriano de Albuquerque, João Vitor do Nascimento Olímpio, Fernanda Jorge Magalhães, Claudinalle Farias Queiroz de Souza, Walmir Soares da Silva Junior

bibliográfico sendo pautados para composição do projeto. De início decidiu-se que as crianças acima de 6 anos e os adolescentes seriam os operadores do jogo, sendo este, feito exclusivamente para eles.

O design do jogo foi elaborado em forma de filme, onde, em sete cenas, o vídeo se transforma em jogo. São divididas em cinco cenas caracterizando a alienação parental e duas outras cenas do jogo se configuram no formato de minijogos. Foram utilizados elementos próprios do Scratch adaptados ao contexto.

$\mathrm{Na}$ fase final, o material foi apresentado aos docentes responsáveis pela equipe para que pudessem contribuir com melhorias para aumentar a qualidade do jogo de acordo com os parâmetros determinados de compatibilidade com a bibliografia, originalidade, efetividade e utilidade. Sucessivamente, o resultado foi exposto pelo grupo para todos os discentes da turma, e docentes envolvidos no módulo, no formato de apresentação online. A explanação foi em através de slides contendo introdução, objetivos, método de pesquisa, elaboração jogo, considerações finais e, por fim, a apresentação do game na plataforma Scratch.

Perante o exposto, exibindo o tipo de estudo, compreende-se que não foi necessária a avaliação pelo sistema CEP/CONEP e foram respeitados os princípios propostos pela resolução $n^{\circ}$ 466/2012, explícito pelo caráter educacional da proposta do estudo.

\section{RESULTADOS}

Os resultados são apresentados através da descrição das cenas e funcionalidades do game. O game está disponível no link: https://scratch.mit.edu/projects/509014039/.

A partir de leitura sobre a temática e visando a ênfase no combate contra a alienação parental, o game "AlieNÃO" foi denominado assim pela junção das palavras "Alienação" e "Não". O jogo foi desenvolvido de forma que o jogador tenha a possibilidade de selecionar as atitudes do personagem principal frente às situações de alienação. Dessa forma, ao final de cada cena, são mostradas 2 (duas) opções de ações, das quais o indivíduo deve clicar naquela que julgar ser a mais correta diante do contexto.

O protótipo do game foi desenvolvido pela construção de uma estória protagonizada por um personagem fictício Gabriel, um garoto de 12 anos e por seus pais que estão em processo de separação conjugal. No decorrer do game, o jogador será responsável por escolher as decisões de conduta para Gabriel, conforme as cenas apresentadas. A depender da opção escolhida, o jogador eleva ou reduz sua barra de pontuação, a qual corresponde seu estado de saúde física e mental, tendo três pontos como pontuação máxima.

Entre as cenas, o jogador tem a possibilidade de acessar dois minijogos, formulados com o objetivo de desafiar ainda mais quem está jogando, além de contribuir para a diversão e descontração durante a partida. O primeiro minijogo consiste em um desafio onde o jogador precisa coletar corações que caem do céu e o segundo é do tipo labirinto. Caso o jogador consiga alcançar os objetivos propostos pelos minijogos, acumulará mais pontos. 


\section{RECIMA21 - REVISTA CIENTÍFICA MULTIDISCIPLINAR ISSN 2675-6218}
JOGO EDUCATIVO PARA A PREVENÇÃO DA ALIENAÇÃO PARENTAL DE CRIANÇAS E ADOLESCENTES Maria Aparecida Beserra, Emily Roberta Gonçalves da Silva, Gabriela Guimarães da Mota, Gabriela Wanderley da Silva, Gabrielle Valeriano de Albuquerque, João Vitor do Nascimento Olímpio, Fernanda Jorge Magalhães, Claudinalle Farias Queiroz de Souza, Walmir Soares da Silva Junior

Considerando o público-alvo, que consiste em crianças a partir dos 6 anos e adolescentes, o game se propõe a atingir os seguintes objetivos educacionais: reiterar a importância do estímulo à autonomia desses indivíduos; apresentar possíveis consequências psicológicas despertadas nos menores devido à alienação parental; estimular a boa relação com os pais ou responsáveis; estimular o bom comportamento e respeito.

$\mathrm{Na}$ Figura 2 observa-se a tela de início, a qual tem seu design composto por tons vibrantes de azul e laranja, de forma a ser atrativo para o público. Na parte superior, o nome do game é apresentado, os personagens principais da narrativa, Gabriel no centro e seus pais ao seu lado, e na lateral esquerda é apresentado o menu com as opções: jogar, regras e sobre.

Figura 2 - Tela de início do jogo "AlieNÃO". Recife, 2021.

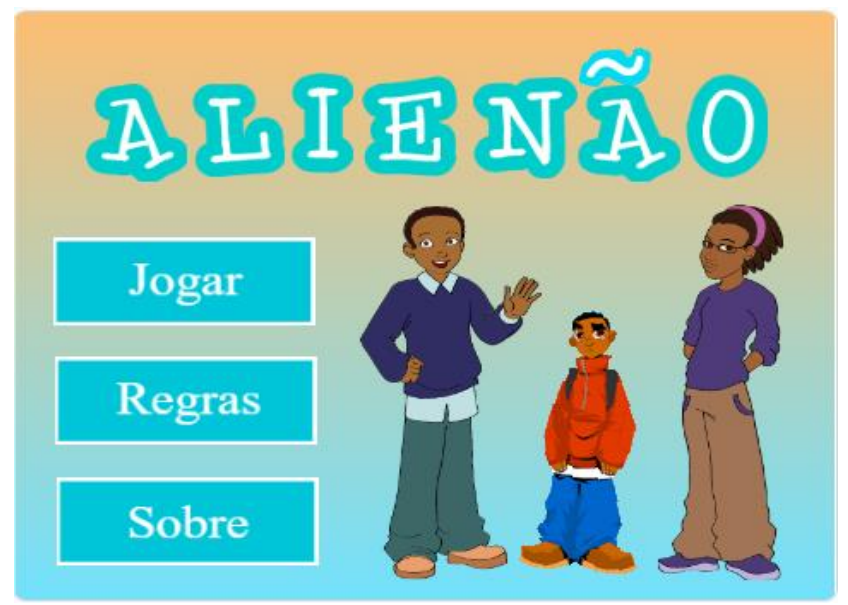

Fonte: Autores.

$\mathrm{Na}$ opção "Regras", o jogador tem a possibilidade de entender com mais detalhes o funcionamento do game através de orientações, as quais são: 1. O game é embasado numa estória de uma família relacionada à Alienação Parental e protagonizada por Gabriel, o filho do casal. Atente-se para cada detalhe do drama; 2. Ao decorrer do game irão surgir situações vivenciadas pela família, na qual o jogador será responsável por selecionar as atitudes do personagem; 3. Lembrem-se que existem rumos viáveis e inviáveis e isso implicará na sua pontuação; 4. Note que a cada cena deverá ser feita uma escolha pelo caminho a ser seguido, nestes, o jogador ganhará ou perderá pontos; 5 . Entre uma cena e outra haverá a opção de o jogador realizar um minijogo com o intuito de aumentar seus pontos (que podem ser no máximo 3); 6. Lembrem-se que o objetivo geral do game é que o jogador sempre opte por soluções positivas e que resolva as situações da melhor forma possível.

$\mathrm{Na}$ opção "Sobre", considerando que a maioria do público-alvo desconhece e/ou possui pouco conhecimento acerca da alienação parental e como ela se manifesta, foi criada uma breve explicação da temática, permitindo que o jogador tenha uma visão geral sobre o conceito como também da lei da alienação parental ( $\left.n^{\circ} 12.318\right)$. 


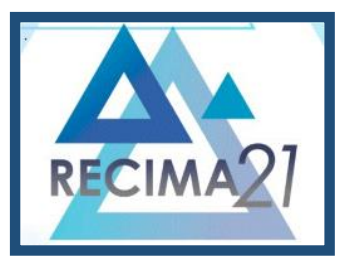

\section{RECIMA21 - REVISTA CIENTÍFICA MULTIDISCIPLINAR ISSN 2675-6218}
JOGO EDUCATIVO PARA A PREVENÇÃO DA ALIENAÇÃO PARENTAL DE CRIANÇAS E ADOLESCENTES Maria Aparecida Beserra, Emily Roberta Gonçalves da Silva, Gabriela Guimarães da Mota, Gabriela Wanderley da Silva, Gabrielle Valeriano de Albuquerque, João Vitor do Nascimento Olímpio, Fernanda Jorge Magalhães, Claudinalle Farias Queiroz de Souza, Walmir Soares da Silva Junior

$\mathrm{Na}$ opção "Jogar", o jogador pode iniciar o game. Ao clicar, o jogador é direcionado para a cena introdutória. Nesta o jogador é introduzido ao personagem principal, Gabriel e a seus pais. Inicialmente é apresentada uma cena com textos que remetem a que todos na família amam uns aos outros, porém, logo a seguir, o cenário muda para o quarto dos pais apresentando uma discussão entre eles. A estória vai sendo contada através de informações e balões de falas dos personagens.

Na cena 1, o primeiro cenário interativo, apresenta Gabriel e a sua mãe fazendo um comentário agressivo sobre o pai de Gabriel. Ao final, são mostradas duas opções: 1. Gabriel vai pedir para a mãe parar de falar mal do pai na frente dele, ou 2. Gabriel não vai falar nada e vai para o seu quarto. Dessa forma, o jogador deve clicar na opção que julgar ser a mais correta diante do contexto. Também observase, na parte inferior da cena, a barra de pontuações, a qual pode diminuir ou aumentar de acordo com a escolha do jogador (Figura 3).

Figura 3 - Primeira cena interativa de escolha do game

"AlieNÃO”. Recife, 2021.

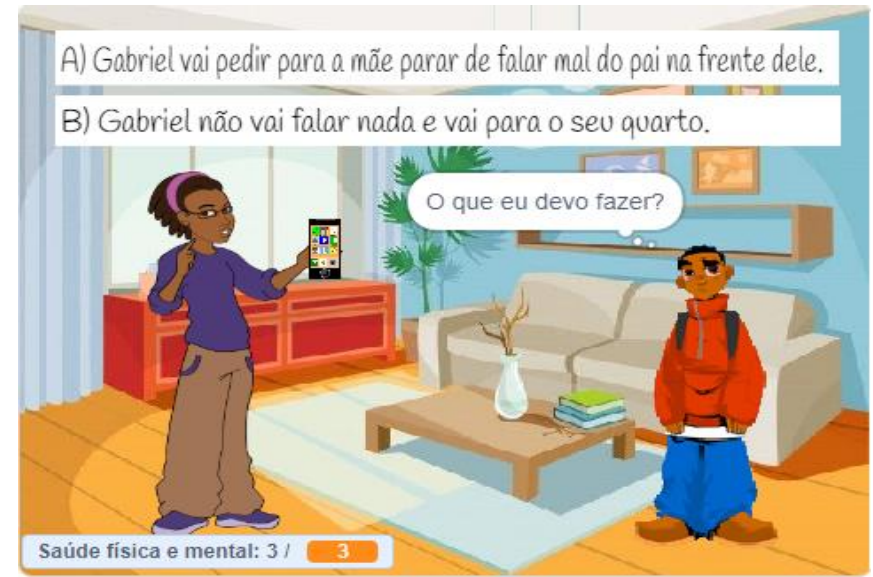

Fonte: Autores.

Após a Cena 1, o jogador é direcionado para a tela inicial do primeiro minijogo Ihe dando a opção de jogar para ganhar pontos ou não. A ideia é trazer um conforto para Gabriel após a situação ocorrida anteriormente. O objetivo deste minijogo é que Gabriel consiga coletar 30 corações inteiros em, no máximo 90 segundos. Caso ele encoste em corações partidos, a quantidade de corações coletados é diminuída. Para que o personagem se movimente e consiga alcançar os corações, deve-se utilizar as setas direita e esquerda do teclado. Observa-se o cenário e elementos do minijogo dos corações. É possível notar a barra de contagem dos corações inteiros coletados juntamente com o marcador de tempo (Figura 4). 


\section{RECIMA21 - REVISTA CIENTÍFICA MULTIDISCIPLINAR ISSN 2675-6218}

JOGO EDUCATIVO PARA A PREVENÇÃO DA ALIENAÇÃO PARENTAL DE CRIANÇAS E ADOLESCENTES Maria Aparecida Beserra, Emily Roberta Gonçalves da Silva, Gabriela Guimarães da Mota, Gabriela Wanderley da Silva, Gabrielle Valeriano de Albuquerque, João Vitor do Nascimento Olímpio, Fernanda Jorge Magalhães, Claudinalle Farias Queiroz de Souza, Walmir Soares da Silva Junior

Figura 4. Minijogo dos corações do game "AlieNÃO".

Recife, 2021.

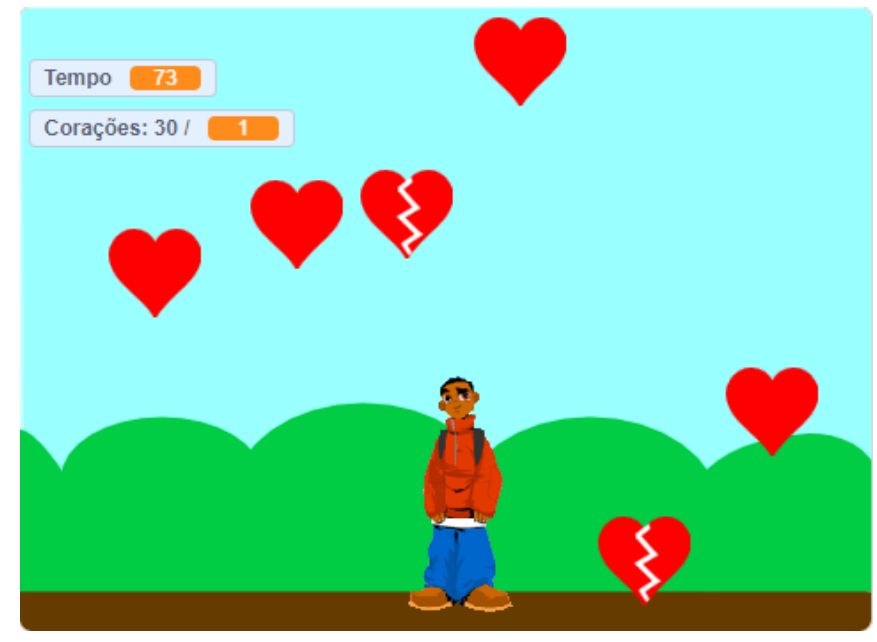

Fonte: Autores.

A segunda cena mostra o pai de Gabriel conversando ao telefone com sua mãe. Ele relata que não pode mais levar o filho para a praia, como tinham combinado, pois precisará trabalhar. Ao final da ligação, Gabriel aparece no quarto da mãe e questiona sobre o que seu pai falou. A mãe conta que o pai não está mais disponível para passear com Gabriel, porém, em vez de explicar que o motivo se deve ao trabalho, ela diz ao filho que seu pai simplesmente não quer vê-lo. Ao término da cena, o jogador deve escolher o que Gabriel deve fazer nessa situação: dizer: "Meu pai não faria isso, quero falar com ele." ou falar: "Ah é?... Também não quero mais vê-lo".

Na cena 3 o pai de Gabriel chega em sua casa para buscá-lo, pois o filho deve passar o fim de semana com ele. O pai entra na casa e a mãe de Gabriel avisa de sua chegada, porém, ao ver seu pai, Gabriel fala de maneira séria: "Não quero ir, quero ficar aqui com a minha mãe!" O pai fica visivelmente triste e pergunta "Certeza? Eu fiz algo que te deixou magoado?". Diante dessa cena, o jogador deve escolher entre as seguintes opções: Gabriel afirma novamente que não quer sair com o pai ou Gabriel questiona o pai sobre as coisas que a mãe fala a respeito dele.

Dando seguimento, após a cena 3, o jogador tem a opção de jogar o segundo minijogo, o qual consiste em um labirinto. Da mesma forma que o primeiro, é feita a seguinte pergunta: "Você deseja jogar um minijogo para aumentar a sua pontuação, fazendo com que Gabriel se sinta melhor?" Se a opção "NÃO" for escolhida, o jogador será direcionado para a próxima e última cena. Caso o "SIM" seja selecionado, o minijogo será iniciado. Como objetivo, Gabriel precisa ser levado até o personagem do seu pai, que encontra-se na saída do labirinto, onde o jogador deve utilizar as setas do teclado (cima, baixo, direita, esquerda) e movimentar o personagem de Gabriel para que ele encontre seu pai em um tempo menor que 100 segundos.

A última cena do game é ambientada na escola de Gabriel. Neste cenário, sua professora pergunta se está tudo bem com ele, pois, tem notado que Gabriel não vem prestando atenção nas aulas e suas 


\section{RECIMA21 - REVISTA CIENTÍFICA MULTIDISCIPLINAR ISSN 2675-6218}

JOGO EDUCATIVO PARA A PREVENÇÃO DA ALIENAÇÃO PARENTAL DE CRIANÇAS E ADOLESCENTES
Maria Aparecida Beserra, Emily Roberta Gonçalves da Silva, Gabriela Guimarães da Mota,
Gabriela Wanderley da Silva, Gabrielle Valeriano de Albuquerque, João Vitor do Nascimento Olímpio,
Fernanda Jorge Magalhães, Claudinalle Farias Queiroz de Souza, Walmir Soares da Silva Junior notas estão diminuindo. São mostradas as seguintes opções de resposta na tela: "Não, professora. Eu estou triste com algumas coisas" e "Sim, professora. Desculpe! Prometo me esforçar para melhorar as minhas notas". Ao término dessa cena 4, o jogador é direcionado para a tela final do jogo. Caso a pontuação de saúde física e mental tenha sido zerada, é declarado que o indivíduo perdeu o jogo, sendo mostrada a mensagem: "Infelizmente, você fez escolhas que prejudicaram a saúde de Gabriel e, por isso, acabou perdendo o jogo". Do contrário, o jogador vence e recebe a seguinte mensagem: "Você terminou o jogo e construiu uma ótima relação entre Gabriel e sua família. Continue assim!".

\section{DISCUSSÃO}

A revisão sistemática realizada apresentou que mesmo com um período longo de 10 anos, a quantidade de artigos sobre o tema é reduzida, podendo assim apresentar a necessidade de realização de estudos e pesquisas nesta temática. Certamente conhecer a legislação é um passo fundamental na construção da cidadania dos menores, em vista disso, dentre todas as regulamentações, a lei de alienação parental foi a escolhida como principal referência no desenvolvimento do game.

O game intitulado "AlieNÃO" é uma estratégia de metodologia ativa que tem viés didáticopedagógico. Como os recursos tecnológicos estão cada vez mais avançados nos últimos anos, a utilização das Tecnologias de informação e Comunicação (TIC) têm influenciado na vivência, comunicação e aprendizado dos indivíduos. Sendo assim, a aplicação da mesma no âmbito da educação em saúde vem se expandindo, uma vez que juntamente a revolução tecnológica, o uso de jogos digitais voltado para fins educacionais, conhecido como Serious Games, está sendo cada vez mais aplicado em áreas de conhecimento distintas (OLIVEIRA, 2016).

Ademais, para Vygotsky (1998) os jogos têm um papel na aprendizagem e no desenvolvimento infantil através da aderência às regras e busca por soluções para as questões que lhe são impostas na vida real, uma vez que quando o indivíduo joga utiliza-se de seus conhecimentos e adquire novos, sendo, portanto, importante no processo de ensino-aprendizagem (CRUZ, 2021).

A junção da educação em saúde com os recursos tecnológicos para criação desse jogo interativo tem como proposta que os indivíduos aprendam a identificar a alienação parental fomentando sua autonomia, sendo assim implementado a educação em saúde estimulando-os a agir conscientemente diante de cada ação do cotidiano melhorando sua qualidade de vida. Durante a pesquisa e elaboração do projeto, foi notório que o ato de aprender está ligado a estímulos motivacionais. Sendo assim, fazer uso do serious games no contexto da educação em saúde torna-se uma estratégia eficaz na promoção da saúde visto que as dinâmicas e brincadeiras são instrumentos inerentes ao processo de ensinoaprendizagem, pois resulta em maior envolvimento e no aprendizado das crianças e dos adolescentes (ALMEIDA et al, 2016).

Além disso, no âmbito da formação do profissional enfermeiro a gamificação contribuirá para que os acadêmicos tenham contato com cenários reais de sua vivência profissional, resultando assim na preparação de profissionais para os desafios da assistência à saúde. Isso torna-se importante uma vez que o profissional de enfermagem, deve ter conhecimento dos direitos da criança e do adolescente, para RECIMA21 - Ciências Exatas e da Terra, Sociais, da Saúde, Humanas e Engenharia/Tecnologia 


\section{RECIMA21 - REVISTA CIENTÍFICA MULTIDISCIPLINAR ISSN 2675-6218}
JOGO EDUCATIVO PARA A PREVENÇÃO DA ALIENAÇÃO PARENTAL DE CRIANÇAS E ADOLESCENTES Maria Aparecida Beserra, Emily Roberta Gonçalves da Silva, Gabriela Guimarães da Mota, Gabriela Wanderley da Silva, Gabrielle Valeriano de Albuquerque, João Vitor do Nascimento Olímpio, Fernanda Jorge Magalhães, Claudinalle Farias Queiroz de Souza, Walmir Soares da Silva Junior

que os direitos fundamentais previstos no ECA sejam garantidos e cumpridos, resultando na prestação de uma assistência mais humanizada e digna a esse público (NEUTZLING et al., 2017).

Espera-se que o jogo "AlieNÃO" contribua no combate à alienação parental, promova autonomia e criticidade nas crianças e adolescentes e propicie aprendizagem acerca da temática. Além do mais, o jogo educativo atua como uma prática viável e de baixo custo para os serviços de saúde, pensando nele como uma forma de aprimorar os cuidados de enfermagem com as crianças e adolescentes.

De acordo com a relevância social da criação de tecnologias com enfoque na promoção da saúde, espera-se que o presente estudo contribua para a difusão do procedimento de jogos educativos como uma opção coerente para o trabalho de análise comportamental. Assim, desenvolve-se um novo olhar na relação entre criança/adolescente e família.

O enfermeiro, em instituições de saúde, é ímpar em analisar comportamentos da criança/adolescente, a fim de diagnosticar maus tratos e/ou negligência. Dessa forma, é trazida a necessidade do estímulo ao acompanhamento profissional para prevenção e diagnóstico de manifestações que ponham em risco a integridade das crianças e adolescentes.

Ainda nessa perspectiva, mas afunilando o debate à saúde e mais especialmente ao papel da enfermagem na garantia e efetivação do direito mencionado, é nítido que é um desafio para os profissionais de enfermagem dessa área de atuação, ficando a cargo deles a identificação de casos de Alienação Parental mediante a consulta de enfermagem e diagnosticando traços do comportamento que sejam prejudiciais para o crescimento e desenvolvimento desses indivíduos.

\section{CONSIDERAÇÕES FINAIS}

Este estudo apresentou uma metodologia atual e inovadora a qual possibilita o uso de jogos educativos pelos enfermeiros como um recurso de intervenção de Enfermagem. Apresentou o tema alienação parenteral, ainda pouco discutido, como forma de apoiar estes profissionais a assistirem de forma mais direcionada na prevenção desse acontecimento e ainda possibilitar às crianças e adolescentes identificarem se estão sendo submetidas a esta situação.

O relato da experiência de docentes e discentes do Curso de Enfermagem neste artigo pretende ainda fomentar a presença de novas tecnologias no ensino de graduação, a fim de formar profissionais com competências e habilidades na área de Informática em Enfermagem.

Como limitação deste estudo, aponta-se a não validação do game por especialistas na área e pelos usuários.

\section{REFERÊNCIAS}

AIRES, J. P. Alienação parental e suas implicações na saúde da criança: uma revisão integrativa. 2018. TCC (Graduação em Enfermagem) - Universidade Federal do Rio Grande do Sul, Porto Alegre, 2018. Disponível em: https://lume.ufrgs.br/handle/10183/193836.

ALMEIDA, E. R.; MOUTINHO, C. B.; LEITE, M. T. S. Prática pedagógica de enfermeiros de Saúde da Família no desenvolvimento da Educação em Saúde. Interface - Comunicação, Saúde, Educação, v. 20, n. 57, p. 389-402. DOI: https://doi.org/10.1590/1807-57622015.0128.

RECIMA21 - Ciências Exatas e da Terra, Sociais, da Saúde, Humanas e Engenharia/Tecnologia 


\title{
RECIMA21 - REVISTA CIENTÍFICA MULTIDISCIPLINAR ISSN 2675-6218
}

\author{
JOGO EDUCATIVO PARA A PREVENÇÃo DA ALIENAÇÃo PARENTAL DE CRIANÇAS E ADOLESCENTES \\ Maria Aparecida Beserra, Emily Roberta Gonçalves da Silva, Gabriela Guimarães da Mota, \\ Gabriela Wanderley da Silva, Gabrielle Valeriano de Albuquerque, João Vitor do Nascimento Olímpio, \\ Fernanda Jorge Magalhães, Claudinalle Farias Queiroz de Souza, Walmir Soares da Silva Junio
}

BEZERRA, T. A.; LOW, S. T.; LIMA T. G.; LINS, V. M. C. D.; MAIA, T. S.; ARAUJO, R. L.; LOPES S. M. S. Software Educativo: ferramenta direcionada para educação em saúde de crianças com Tetralogia de Fallot. Revista de Enfermagem Digital Cuidado e Promoção da Saúde, v. 4, n. 2, p. 134-139, 2019. Disponível em: https://cdn.publisher.gn1.link/redcps.com.br/pdf/v4n2a10.pdf.

CASTRO, T. C.; GONÇALVES, L. S. The use of gamification to teach in the nursing field. Revista Brasileira de Enfermagem, v. 71, n. 3, p. 1038-1045, 2018. Disponível em: https://doi.org/10.1590/00347167-2017-0023.

COLUCCI NETO, V.; MARTINS, C. C. Saúde psíquica infanto-juvenil em casos de alienação parental: uma reflexão jurídica e psicológica. Archives of Health Investigation, v. 8, n. 4, 2019. DOI: https://doi.org/10.21270/archi.v8i4.4698.

CRUZ, L. A. M.; LOPO, A. B. Jogos digitais no ensino de geometria: desenvolvimento de um quiz. Revista Scientia, $\quad$ v. $6, \quad$ n. $2, \quad$ p. 22-40, 2021. Disponível em: https://www.revistas.uneb.br/index.php/scientia/article/view/10124/7835.

LIMA, A. B. Alienação parental - um jogo cruel para crianças. [S. I.]: Jus, 2018. Disponível em: https://jus.com.br/artigos/70354/alienacao-parental-um-jogo-cruel-para-criancas.

MONTEZUMA, M. A.; PEREIRA, R. C.; MELO, E. M. Abordagens da alienação parental: proteção e/ou violência? Physis: Revista de Saúde Coletiva, v. 27, n. 4, p. 1205-1224, 2017. DOI: https://doi.org/10.1590/S0103-73312017000400018.

NEUTZlinG, B. R. S.; BARleM, J. G. T.; BARLEM, E. L. D.; HIRSCH, C. D.; PEREIRA, L. A.; SCHALLENBERGUER, C. D. Em defesa dos direitos da criança no ambiente hospitalar: o exercício da advocacia em saúde pelos enfermeiros. Escola Anna Nery, v. 21, n. 1, e20170025, 2017. DOI: https://doi.org/10.5935/1414-8145.20170025.

OLIVEIRA, A. E. F. Serious games na educação em saúde. Jornal Brasileiro de Telessaúde, v. 4, n. 2, p. 1-2, 2016. $\quad$ Disponível em: https://www.epublicacoes.uerj.br/index.php/jbtelessaude/article/view/33528/23774.

PORTO, R. T. C.; DUPONT, F. R. A Participação da criança desde a primeira infância nas ações que visem enfrentar a violência intrafamiliar e suas consequências como uma estratégia fundamental para a construção de uma política efetiva. Revista Barbarói, v. 1, n. 47, p. 179-192, 2016. DOI: http://dx.doi.org/10.17058/barbaroi.v0i47.9563.

PAGE, M.; MACKENZIE, J. E.; BOSSUYT, P. M.; BOUTRON, I.; HOFFMANN, T. C.; MULROW, C. D.; SHAMSEER, L.; TETZLAFF, J. M.; AKL, E. A.; BRENNAN, S. E.; CHOU, R.; GLANVILLE, J.; GRIMSHAW, J. M.; HRÓBJARTSSON, A.; LALU, M. M.; LI, T.; LODER, E. W. MAYO-WILSON, E.; MCDONALD, S.; MOHER, D. A declaração PRISMA 2020: uma diretriz atualizada para relatar revisões sistemáticas. BMJ, v. 372, n. 71, 2021. DOl https://doi.org/10.1136/bmj.n71

POSSOLLI, G.; MARCHIORATO, A.; NASCIMENTO, G. Gamificação como recurso educacional na área da saúde: uma revisão integrativa. Educação \& Tecnologia, v. 23, n. 3, 2020. Disponível em: https://seer.dppg.cefetmg.br/index.php/revista-et/article/view/783

SILVA, P. S. C. Modelos de aplicação da gamificação na saúde pública. In.: TOLEDO, A. G. L.; KÄHLER, P. Y. (Orgs.). Empreendedorismo e inovação na gestão pública. Campo Grande: Inovar, 2019. p. 7994. 